КЛИНИКО-БИОХИМИЧЕСКАЯ ХАРАКТЕРИСТИКА ПАЦИЕНТОВ С ПЕРВИЧНЫМ БИЛИАРНЫМ ЦИРРОЗОМ ПЕЧЕНИ ${ }^{1}$ Е. Г. Шевченко (shauchenka.bazhko.alena@gmail.com), 23. П. Лемешевская (lemeshevskayazp@tut.by), ${ }^{2}$ Н. И. Прокопчик ${ }^{1}$ УО «Белорусский государственный медицинский университет», Минск, Беларусь, 2УО «Гродненский государственный медицинский университет», Гродно, Беларусь

Введение. Первичный билиарный цирроз (ПБЦ) - редкое аутоиммунное заболевание, характеризующееся медленным прогредиентным течением воспалительного процесса в портальных трактах и иммуноопосредованной деструкцией внутрипеченочных желчных протоков.

Цель исследования - представить клинико-лабораторную характеристику пациентов с ПБЦ в Республике Беларусь.

Материалы и методы. Объектом исследования был 71 пациент с диагнозом ПБЦ. Материалом для исследований служили: плазма и сыворотка крови пациентов, биопсийный материал печени, данные теста связывания чисел. Качество жизни определялось при поступлении с помощью Short Form Medical Outcomes Study (SF-36).

Результаты. Все пациенты с ПБЦ - женского пола, средний возраст - 55 лет, что совпадает с климактерическим периодом. Представлена клинико-лабораторная характеристика и проведен анализ психоэмоционального статуса пациентов с ПБЦ.

Заключение. В статье дано описание пациентов с ПБЦ с учетом клинико-биохимической характеристики и возраста. Владение этой инфрормацией позволит на более ранних стадиях выявлять данную патологию, проводить профрилактику развития терминальной стадии заболевания, что ведет к уменьшению влияния прогрессивного характера болезни.

Ключевые слова: первичный билиарный цирроз, клинические синдромы, лабораторная диагностика, качество жизни.

Для цитирования: Шевченко, Е. Г. Клинико-биохимическая характеристика пациентов с первичным билиарным циррозом печени / Е. Г. Шевченко, З. П. Лемешевская, Н. И. Прокопчик // Гепатология и Гастроэнтерология. 2019. T. 3, № 1. C. 49-54. https://doi.org/10.25298/2616-5546-2019-3-1-49-54

\title{
CLINICAL AND BIOCHEMICAL CHARACTERISTICS OF PATIENTS WITH PRIMARY BILIARY CIRRHOSIS
}

${ }^{1}$ E. G. Shauchenka, ${ }^{2 Z}$. P. Lemeshevskaya, ${ }^{2}$ N. I. Prokopchik ${ }^{1}$ Educational institution «Belarusian state medical University», Minsk, Belarus ${ }^{2}$ Educational institution «Grodno state medical University», Grodno, Belarus

Background. Primary biliary cirrhosis $(P B C)$ is a rare autoimmune disease characterized by a slow progredient course of the inflammatory process in the portal tracts and immune-mediated destruction of the intrahepatic bile ducts.

Objective: to describe clinical and laboratory characteristics of patients with primary biliary cirrhosis in the Republic of Belarus.

Materials and methods. The subjects of the study were 71 patients diagnosed with PBC. The materials of the study were patients' blood plasma and serum, bioptic liver specimens, and data of the number connection test. Quality of life was assessed on admission using Short Form Medical Outcomes Study (SF-36).

Results. All patients with PBC were female; the average age was 55 years, that coincides with menopause. The article presents clinical, laboratory characteristics and the analysis of psycho-emotional status of patients with PBC.

Conclusion. The article describes patients with PBC taking into account clinical and biochemical characteristics and age. These data will contribute to earlier detection, prevention of end-stage disease and the improvement of associated symptoms thus reducing the impact of the progressive nature of the disease.

Keywords: primary biliary cirrhosis, clinical syndromes, laboratory diagnostics, quality of life.

For citation: Shauchenka EG, Lemeshevskaya ZP, Prokopchik NI. Clinical and biochemical characteristics of patients with primary biliary cirrhosis . Hepatology and Gastroenterology. 2019;3(1):49-54. https://doi.org/10.25298/2616-5546-2019-3-1-49-54

\section{Введение}

Первичный билиарный цирроз (ПБЦ) - редкое аутоиммунное заболевание, характеризующееся медленным прогредиентным течением воспалительного процесса в портальных трактах и иммуноопосредованной деструкцией внутри- печеночных желчных протоков [1]. ПБЦ встречается во всех частях мира среди людей всех рас и национальностей без каких-либо различий по географичческому признаку [2]. Средняя распространенность ПБЦ составляет 40-50 случаев на 1 млн взрослого населения. Наиболее часто ПБЦ 
развивается в возрасте от 40 до 50 лет и крайне редко - у людей моложе 25 лет, диагностируется преимущественно у женщин (соотношение мужчин и женщин - 6:10) среднего возраста (35-60 лет) и может иметь семейный характер [3].

Цель исследования - представить клинико-лабораторную характеристику пациентов с ПБЦ в Республике Беларусь.

\section{Материалы и методы}

Объектом исследования были пациенты (71 чел.) с диагнозом ПБЦ, проходившие курс стационарного лечения в УЗ «Минская областная клиническая больница», УЗ «Городская клиническая больница № 6 г. Минска», УЗ «Городская клиническая больница № 4 г. Гродно». Исследование длилось в период с 2009 по 2016 гг.

Исходное обследование пациентов проводили в период обострения заболевания при поступлении в стационар. Всем пациентам выполняли общеклинические исследования согласно протоколам диагностики и лечения МЗ РБ. Пациенты с ПБЦ, включенные в исследование, прошли собеседование и подписали лист информированного согласия, соответствующий принципам биомедицинской биоэтики.

Критерием включения пациентов в исследование было наличие соответствия критериям нозологической формы - ПБЦ, представленных в клинических протоколах диагностики в соответствии с Международной классификацией болезней ВОЗ (МКБ-10) и коду К74.3.

Клиническими критериями ПБЦ считали: кожный зуд, слабость, синдром Шегрена, ксантелазмы и ксантомы, сосудистые стигматы, спленомегалию, желтуху, портальную гипертензию.

Материалом для исследований служили: плазма и сыворотка крови пациентов, биопсий- ный материал печени, данные теста связывания чисел.

Лабораторные показатели были представлены: общим анализом крови, включая тромбоциты и ретикулоциты, биохимическим анализом крови (билирубин, АсАТ, АлАТ, ЩФ, ГГТП, альбумин, глюкоза, холестерин - ХС, мочевина, креатинин, общий белок, альбумин, ПТИ или МНО), общий анализ мочи. Одновременно исследовались специфические для аутоиммунного процесса маркеры (аутоантитела): ANA, AMA или AMA-2M, SMA, anti-LKM (при высоком уровне АсAТ, АлАТ), содержание IgM. Для исключения вирусной этиологии проводили обследование на маркеры вирусных гепатитов (HBsAg, anti-HCV). Всем пациентам выполнена сонография органов брюшной полости и ФГДС.

Диагностическими критериями ПБЦ считали уровень ЩФ более двух норм или ГГТП более 5 норм; титр AMA >1:40; по данным биопсии печени: деструкция желчных протоков (рис. 1, 2, $3,4,5)$ (биопсия не обязательна при наличии первых двух критериев, но позволяет оценить активность и стадию заболевания; необходима для диагноза при отсутствии специфических аутоантител), а также использовали результаты Fibro Test (рис. 6).

C помощью метода стратифицированной рандомизации пациенты были разделены на группы, в которых проводилась в последующем дифрференцированная или стандартная терапия.

Качество жизни определялось при поступлении с помощью Short Form Medical Outcomes Study (SF-36). Применялся опросник SF-36 и его онлайн-калькулятор. Все 36 пунктов опросника были сгруппированы в восемь шкал: физическое функционирование, ролевая деятельность, телесная боль, общее здоровье, жизнеспособ-
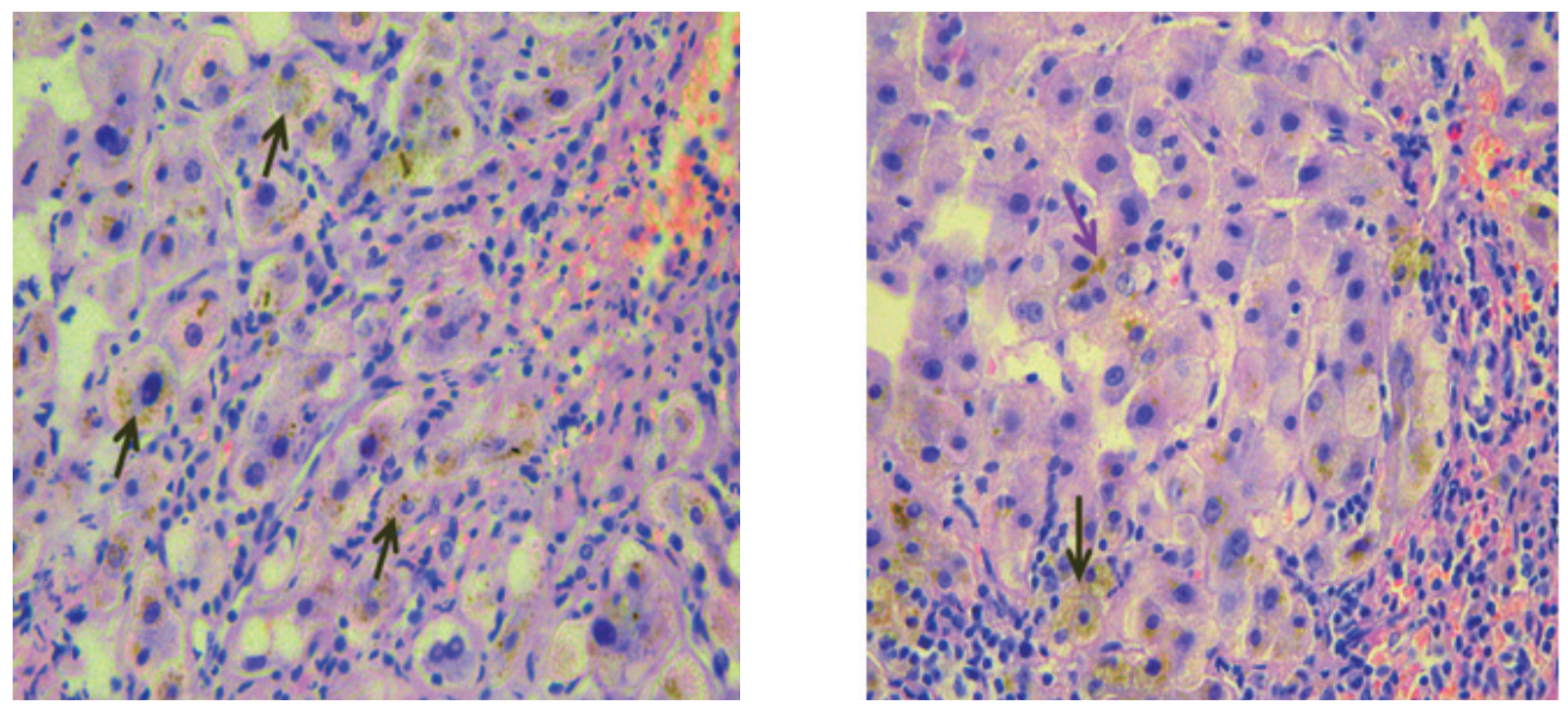

Рисунок 1. - ПБЦ: балочное строение печени нарушено, дискомплексация гепатоцитов, резко выраженный внутридольковый холестаз (внутриклеточный - черные стрелки, и каналикулярный - сиреневая стрелка); белковая дистрофия гепатоцитов. Окр.: гематоксилином и эозином. ×400 

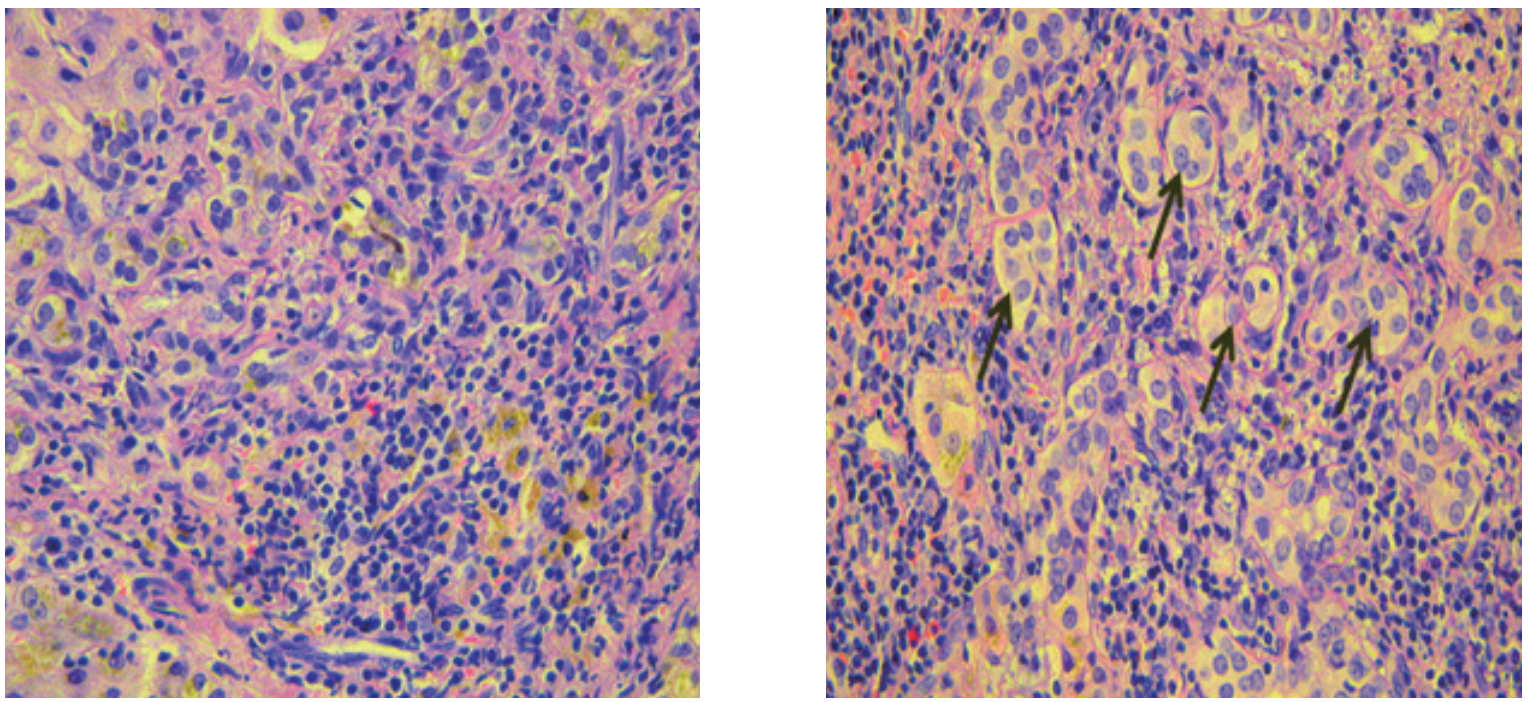

Рисунок 2. - ПБЦ: портальный тракт расширен за счет резко выраженной лимфоидно-гистиоцитарной и плазмоцитарной инфильтрации и фиброза; деструкция и резко выраженная пролифрерация междольковых желчных протоков (стрелки). Пролиферирующие холангиолы занимают территорию всего портального тракта и с клетками воспалительного инфильтрата распространяются в перипортальные отделы долек. Окр.: гематоксилином и эозином. ×400
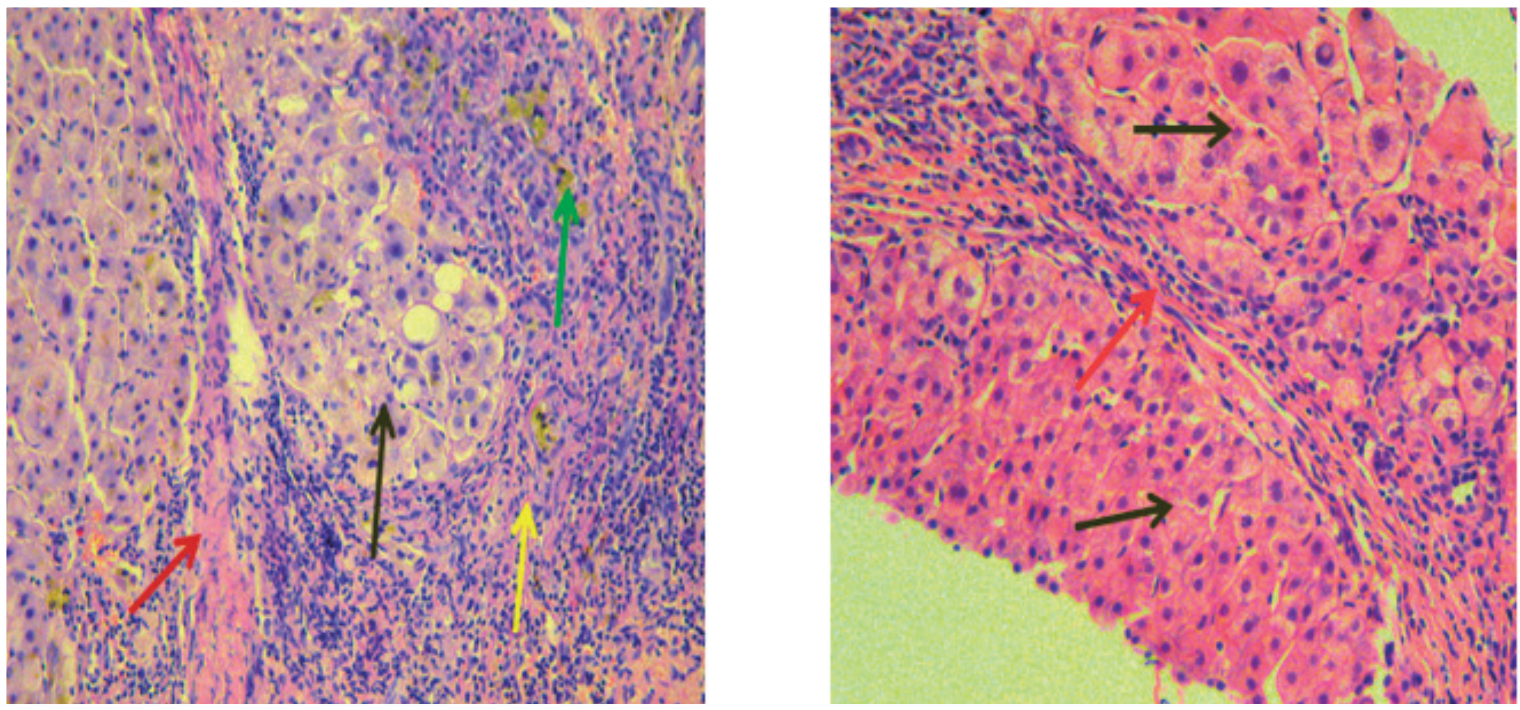

Рисунок 3. - ПБЦ: ложные дольки (черные стрелки), окруженные фиброзной тканью; фриброзные порто-портальные септы (красные стрелки); резко выраженная лимфоидно-гистиоцитарная инфильтрация и склероз портального тракта (желтая стрелка); холестаз (зеленая стрелка); полиморфизм ядер гепатоцитов. Окр.: гематоксилином и эозином. ×200

ность, социальное функционирование, эмоциональное состояние и психическое здоровье. Показатели каждой шкалы варьируют между 0 и 100, где 100 представляет полное здоровье, все шкалы формируют два показателя: душевное и фризическое благополучие. Результаты представляются в виде оценок в баллах по 8 шкалам, составленным таким образом, что более высокая оценка указывает на более высокий уровень качества жизни пациента.

Из исследования исключались пациенты с сопутствующими заболеваниями, которые могли бы исказить результаты обследования: с сахарным диабетом (при условии, что вначале выставлен диабет, а затем - ПБЦ), с заболева- ниями крови, артериальной гипертензией (АГ) III степени (или наличием частых кризов), инфарктами миокарда, явными признаками хронической сердечной недостаточности (XCHIIA, IIБ, III ст.); с системными болезнями соединительной ткани (ревматоидным артритом, системной красной волчанкой); органическими поражениями ЦНС с нарушениями мозгового кровообращения в анамнезе; тяжелыми травмами, инфекционными заболеваниями; оперативными вмешательствами; тяжелыми психическими заболеваниями или психическими и поведенческими нарушениями вследствие употребления психоактивных лекарственных средств; с низкой приверженностью к лечению. Испытуемые с терминальной стадией 


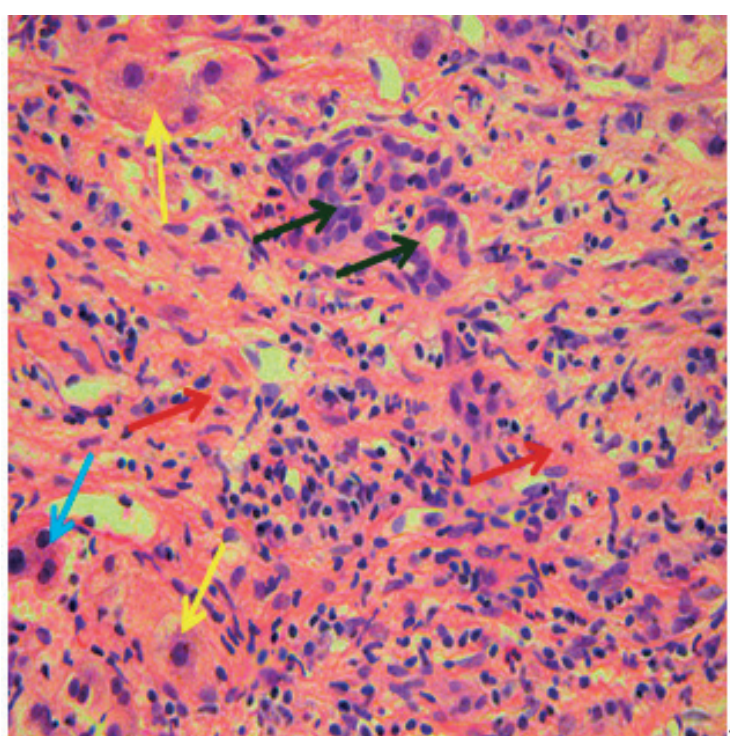

Рисунок 4. - ПБЦ: резко выраженный фиброз портального тракта (красные стрелки), умеренно выраженная лимфоидно-гистиоцитарная и плазмоцитарная инфильтрация: пролиферация желчных протоков (черные стрелки); секвестрация гепатоцитов (желтые стрелки); патологическая регенерация гепатоцита с наличием в цитоплазме 3 ядер (синяя стрелка). Окр.: гематоксилином и эозином. $\times 400$

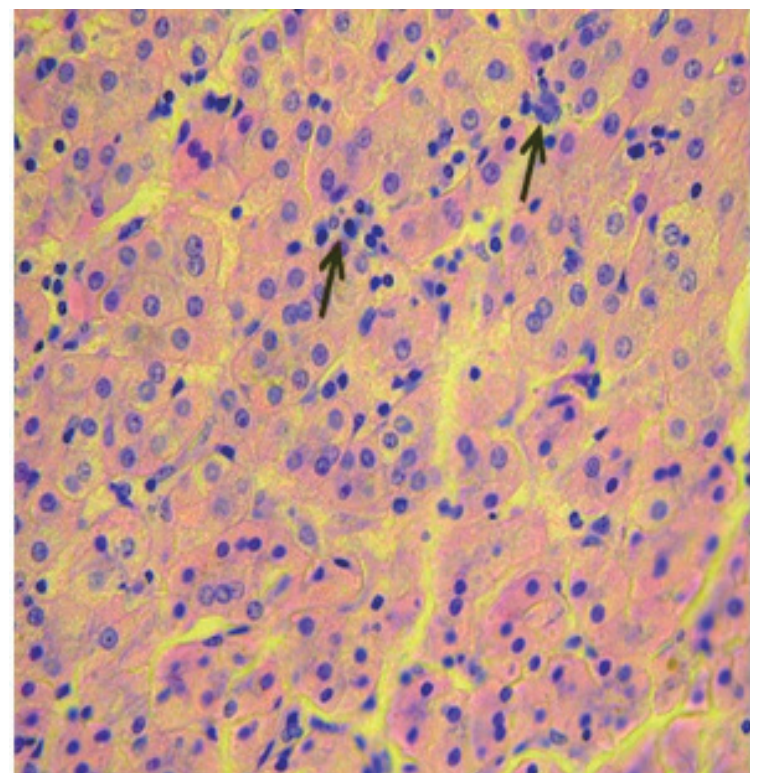

Рисунок 5. - ПБЦ: лимфоидно-гистиоцитарная и плазмоцитарная инфильтрация внутри долек выражена слабо (стрелки); белковая дистрофия гепатоцитов. Окр.: гематоксилином и эозином. $\times 400$

исключались из исследования (если наблюдались прогрессирующая печеночная недостаточность, портальная гипертензия и другие осложнения цирроза печени).

Для объективизации выраженности клинических показателей помимо регистрации имеющейся клинической симптоматики использовали оценку по ориентировочной шкале ВАШ, данные оценивали количественно - это расположенная на листе бумаги вертикальная или горизонтальная линия длиной 100 мм с обозначениями край-

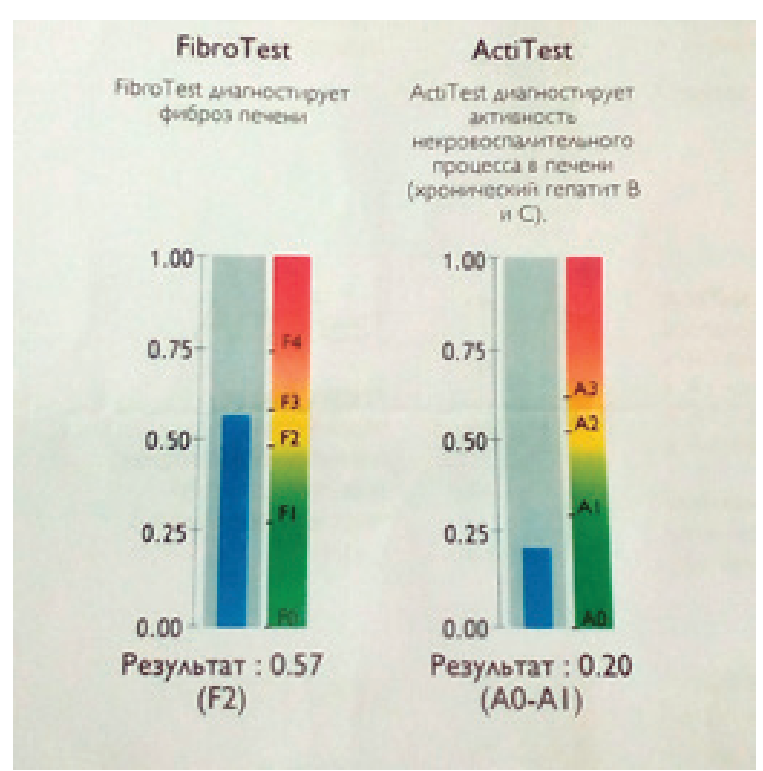

Рисунок 6. - Fibro Test на примере наиболее часто встречающегося результата в исследуемой группе ПБЦ

них ощущений. Надписи на шкале не стандартизованы, но чаще использовали такие фразы, как «совсем нет симптома», «сильный симптом». Для оценки тяжести измеряли расстояние от самой нижней точки шкалы (или от левого ее края при горизонтальном расположении) до точки, указанной пациентом, и проводили статистическую обработку.

Для обработки полученных данных использовали программу Stats Direct и методы непараметрической статистики в связи с тем, что полученные данные не подчинялись нормальному распределению. Для анализа количественных данных использовали U-критерий Манна-Уитни, для анализа качественных показателей использовали таблицы сопряженности и критерий $\mathrm{X}^{2}$ (с поправкой Иейтса при степени свободы U=1) при значении более 5 , при значении менее 5 использовали точный критерий Фишера, для сравнения нескольких групп - критерий Н Крускала-Уоллиca $(p<0,05)$. Применяли корреляцию Спирмена для измерения направления и силы связи между двумя показателями (силу связи считали слабой, если $r \leq 0,25$, средней - $r=0,26-0,75$, сильной $-r \geq 0,76)$ ( $p$ - сравнение с таблицей критических значений).

Результаты представлены в виде Me (25; 75). Групповые различия описаны в абсолютных величинах, если отрицательный тест W Шапиро-Уилка, p>0,05, или в перцентилях при положительном тесте W Шапиро-Уилка, $p<0,001$. Данные опросников, выраженные в балльном эквиваленте, относили к интервальным шкалам. Результаты интервальных шкал и шкал соотношения представили в виде Ме $(25 ; 75)$. Для анализа связи использованы такие показатели непараметрической статистики, как критерий Манна-Уитни, Крускала-Уоллиса, Вилкоксона, 
наравне с $\mathrm{X}^{2}$, критерием Фишера и построением таблиц сопряженности. Кроме того, для анализа результатов в шкалах, где вариантов значений 20 и более, использовали показатели непараметрической статистики анализа количественных данных.

\section{Результаты и обсуждение}

Обследован 71 пациент в возрасте от 18 до 65 лет, среди которых все (100\%) были представлены женским полом. Средний возраст составил 55 (49: 61) лет, продолжительность бо-

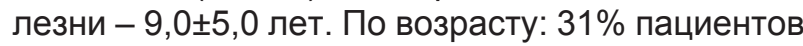
в возрасте до 40 лет, 54\% - от 41 до 50 лет и 15\% - старше 50 лет.

Результаты клинического мониторинга (табл. 1) позволили установить у 13\% пациентов бессимптомную стадию, у большинства - 3 стадию, имеющую клинические проявления и лабораторные признаки ПБЦ ( $22=29,27, p<0,0001)$.

Среди наблюдаемых преобладали пациенты с классом тяжести цирроза по Чаилд-Пью А и В $\left(X^{2}=21,28, p<0,0001\right)$.Средняя частота госпитализаций за год составила 3. Всем пациентам в динамике проводились: оценка выраженности имеющихся симптомов, психоэмоциональных нарушений.

При поступлении у всех (100\%) пациентов с ПБЦ не выявлено признаков асцита клинически и при ультразвуковом исследовании органов брюшной полости.

Среди признаков портальной гипертензии у большинства пациентов по результатам ФГДС обнаруживалось варикозное расширенные вен пищевода $x^{2} 64,8 p<0,0002$.

Наиболее частым кожным проявлением ПБЦ была гиперпигментация (27\%). Изначально потемнение кожи наблюдалось на сгибательных поверхностях конечностей, а впоследствии распространялось на все тело, в то время как ксантелазмы наблюдались только у $7 \%$ пациентов, а желтуха - у 11\%, х 78,5, p<0,0001.

Наиболее типичным клиническим проявлением ПБЦ был кожный зуд, который имелся у $76 \%$ наших пациентов как симптом манифестации заболевания, $x^{2} 43,7, p<0,001$.
Со стороны верхних отделов ЖКТ наблюдался синдром Шегрена, характеризующийся у $64 \%$ пациентов синдромом выраженного холестаза, $\mathrm{X}^{2} 41,4, p<0,0001$. Проявления синдрома Шегрена были слабовыраженными и нераспознанными, так как в субъективной симптоматике болезни доминировал интенсивный кожный зуд (наличие синдрома подтверждено детальным сбором анамнеза заболевания).

Отмечено, что у $18 \%$ пациентов имелись изменения состояния ротовой полости и зубов. Это сопровождалось разрушением твердой пластины, расшатыванием и выпадением зубов, прогрессированием кариеса, что вынуждало пациентов чаще обращаться к стоматологам для протезирования, $x^{2} 43,6, p<0,002$.

У $31 \%$ пациентов практически в одно и то же время с установлением диагноза ПБЦ были верифицированы сахарный диабет или нарушение толерантности к глюкозе.

Поражение легких в виде диффрузного пневмосклероза, который был представлен деформацией легочного рисунка, тяжистыми, ячеистыми тенями на рентгенограммах легких, наблюдалось у $40 \%$ пациентов, на что не было обращено должное внимание лечащими врачами.

Психометрическое тестирование - тест связывания чисел составил 27 (19:48) секунд, что было в пределах нормы для данной возрастной категории.

По шкале «Физическое функционирование» (Physical Functioning - PF), отражающей степень, в которой физическое состояние ограничивает выполнение физических нагрузок (самообслуживание, ходьба, подъем по лестнице, переноска тяжестей и т. п.), показатель составил для ПБЦ - 26 (18:46), p=0,0001 в сравнении с популяционной нормой здоровых.

Достоверной разницы по шкале «Ролевое функционирование», обусловленное фризическим состоянием (Role-Physical Functioning - RP), - влияние физического состояния на повседневную ролевую деятельность (работу, выполнение повседневных обязанностей), не выявлено - 58 $(38: 76), p=0,09$.

Таблица 1. - Распределение пациентов с ПБЦ по стадиям и клиническим проявлениям

\begin{tabular}{|c|c|c|c|c|c|c|c|}
\hline \multicolumn{2}{|c|}{ Показатель } & \multicolumn{2}{|c|}{$\begin{array}{c}18-40 \text { лет, } \\
(\mathrm{n}=22)\end{array}$} & \multicolumn{2}{|c|}{$41-50$ лет, $(n=38)$} & \multicolumn{2}{|c|}{ 51-65 лет, $(n=11)$} \\
\hline \multirow[t]{2}{*}{ Клиническая стадия ПБЦ (n) } & бессимптомная & 5 & - & 2 & - & 2 & - \\
\hline & симптоматическая & - & 17 & - & 36 & - & 9 \\
\hline \multirow{2}{*}{\multicolumn{2}{|c|}{$\begin{array}{l}\text { Стадия цирроза печени, } \\
\text { по Чаилд-Пью (n)19 }\end{array}$}} & A & $\mathrm{B}$ & A & $\mathrm{B}$ & A & $\mathrm{B}$ \\
\hline & & 19 & 11 & 11 & 27 & 4 & 7 \\
\hline \multicolumn{2}{|c|}{ ВРВ пищевода, по данным ФГДС (n) } & \multicolumn{2}{|c|}{17} & \multicolumn{2}{|c|}{36} & \multicolumn{2}{|c|}{9} \\
\hline \multicolumn{2}{|l|}{ Симптом кожного зуда (n) } & \multicolumn{2}{|c|}{16} & \multicolumn{2}{|c|}{31} & \multicolumn{2}{|c|}{7} \\
\hline \multicolumn{2}{|l|}{ Синдром Шегрена (n) } & \multicolumn{2}{|c|}{3} & \multicolumn{2}{|c|}{40} & \multicolumn{2}{|c|}{2} \\
\hline
\end{tabular}


Таблица 2. - Биохимическая и иммунологическая характеристика пациентов с ПБЦ с учетом возраста

\begin{tabular}{|c|c|c|c|}
\hline Показатель & $18-40$ лет, $(n=22)$ & 41-50 лет, $(n=38)$ & 51-65 лет, $(n=11)$ \\
\hline Титр AMA 1:40 (n) & 19 & 34 & 9 \\
\hline Титр по данным ИФА АМА-2M >200 U/ml (n) & 6 & 12 & 4 \\
\hline ANA (индекс антител), >1 (n) & 1 & 3 & 2 \\
\hline Fibro Test стадия F2 и более (n) & 10 & 27 & 6 \\
\hline Уровень щелочной фосфатазы, Ед/л & 295 & 480 & 314 \\
\hline Уровень ГГТП, Ед/л & 310 & 472 & 326 \\
\hline Уровень холестерина, ммоль/л & 5,9 & 8,4 & 6,3 \\
\hline
\end{tabular}

Интенсивность боли (Bodilypain - BP) и еe влияние на способность заниматься повседневной деятельностью, включая работу по дому и вне дома, достоверно больше влияла на качество жизни пациентов с ПБЦ, которые не разделяли понятие боли и наличие мучительного зуда кожи: показатель 32 (37:49), p=0,0003.

Общее состояние здоровья (General Health $\mathrm{GH})$ - оценка пациентом своего состояния здоровья в настоящий момент и перспектив лечения - имело ту же тенденцию: ПБЦ - 32 (26:30), $\mathrm{p}<0,001$.

Жизненная активность (Vitality - VT), подразумевающая ощущение себя полным сил и энергии, или, напротив, обессиленным, при ПБЦ составила 72 (66:81), p=0,08.

Показатель социального функционирования (Social Functioning - SF), определяемый степенью, в которой фризическое или эмоциональное состояние ограничивает социальную активность (общение), не различался статистически у пациентов с ПБЦ и здоровых, составив 47 (46:52), $\mathrm{p}<0,07$.

Ролевое функционирование, обусловленное эмоциональным состоянием (Role Emotional $\mathrm{RE})$, предполагающее оценку степени, в которой эмоциональное состояние мешает выполнению работы, или другую повседневную деятельность, включая большие затраты времени, уменьшение объема работы, снижение ее качества и т. п., были достоверно ниже у пациентов с ПБЦ - 27 (26:35), $p<0,0009$.

Психическое здоровье (Mental Health - MH), характеризующее настроение, наличие депрессии, тревоги, общий показатель положительных эмоций, при ПБЦ составил 26 (25:47), p<0,00001.

Показатели гемограммы и биохимические данные показали: наличие у пациентов с ПБЦ гемоглобина - $140(134: 147)$ г/литр, тромбоцитов - в пределах нормы, $179(161: 415)^{\star} 109$. Уровень билирубина составил 76 (24:125) мкмоль/л, активность АсАТ - 74 (48:230) Ед/л, АлАТ - 87 (47:185) Ед/л, ЩФ - 353 (237:595) Ед/л, ГГТП 338 (160: 556) Ед/л, холестерин - 6,2 (5,3: 9,1) ммоль/л, альбумин - 37 (33:48) г/л, ПТИ - 0,75 $(0,62: 1,04)$ (табл. 2).

\section{Заключение}

Популяция пациентов с ПБЦ в Республике Беларусь представлена в основном женщинами. Наиболее распространенная возрастная группа - в возрасте от 40 до 50 лет. Самое частое клиническое проявление - зуд кожи, что соответствует данным мировой литературы, а самая частая стадия, на которой выявлялось заболевание - клиническая, что доказывает необходимость дальнейшей работы для максимально ранней диагностики заболевания и приведет к своевременному началу лечения, а также улучшению качества жизни пациентов с ПБЦ.

\section{References:}

1. Jones DE, Donaldson PT. Genetic factors in the pathogenesis of primary biliary cirrhosis. Clin. Liver Dis. 2003;7(4):841-864.

2. Parikh-Patel A, Gold EB, Worman H, Krivy KE, Gershwin ME. Risk factors for primary biliary cirrhosis in a cohort of patients from the United States. Hepatology. 2001;33(1):1621. doi: 10.1053/jhep.2001.21165.
3. Bittencourt $\mathrm{PL}$, Farias $\mathrm{AQ}$, Abrantes-Lemos $\mathrm{CP}$, Goncalves LL, Goncalves PL, Magalhães EP, Carrilho FJ, Laudanna AA, Cançado EL. Prevalence of immune disturbances and chronic liver disease in family members of patients with primary biliary cirrhosis. J. Gastroenterol. Hepatol. 2004;19(8):873-878. doi: 10.1111/j.14401746.2004.03396.x 